\title{
Les femmes et le langage selon Charles Bally: "des moments de décevante inadvertance"?
}

\author{
Sylvie Durrer (Lausanne)
}

\section{Abstract: \\ Les femmes et le langage selon Charles Bally: "des moments de décevante inadvertance"?}

Les problématiques rassemblées parfois sous la désignation de "Language and Gender" figurent également dans les publications du linguiste suisse Charles Bally. Cet axe de réflexion, qui n'a guère été remarqué dans son œuvre, mérite d'être mis en lumière, non pas pour faire de Bally un féministe avant l'heure, mais simplement, parce qu'il nous semble important de documenter l'histoire d'une problématique en plein essor. Cet article a donc pour objectif de montrer que la question du traitement des femmes et du féminin par la langue française suscite de nombreuses réflexions dès la fin du XIXe. Il s'agira de procéder à une histoire critique d'un épisode de la "linguistique générique", afin de voir si les actuelles thématiques de cette orientation sont abordées, sous quels aspects, dans quels contextes, avec quels résultats, etc. On pourrait alors être surpris-e de découvrir que Charles Bally n'était pas un cas isolé, mais qu'un grand nombre de contemporains (Nyrop, Brunot, Vendryès, Damourette \& Pichon, Dauzat, etc.), se sont eux aussi interrogés, plus ou moins longuement, sur les rapports entre les femmes, le féminin et le langage. Les réflexions "génériques" de Bally et de ses confrères ne sauraient donc être regardées comme de simples "moments décevante inadvertance".

Car la souffrance met au front de toute femme

Une auréole d'or, ainsi qu' aux purs martyrs (Charles Bally 1895, Poème) $)^{1}$

Depuis quelques années nombreux sont ceux et celles qui s'intéressent à l'œuvre du linguiste suisse Charles Bally (1865-1947), qui fut le premier éditeur du Cours général de linguistique de Saussure et qui lui succéda à l'Université de Genève en 1913. Charles Bally tend à devenir une espèce de référence fédératrice, attirant un grand nombre de chercheurs et de chercheuses venant d'horizons théoriques parfois fort différents : littérature, stylistique, linguistique,

\footnotetext{
1 Fragment d'un poème inédit de Bally, présenté par Hellmann 1988.
} 
sociologie, psychanalyse, etc. Il y a là un paradoxe dans la mesure où Bally - injustement méconnu au temps du développement des théories énonciatives - est reconnu au moment où une grande partie de la linguistique privilégie la dimension interlocutive et conversationnelle; rappelons que le linguiste genevois proposait avant tout une linguistique de l'énonciation, de l'allocution pourrait-on dire, dont il fut même l'un des principaux inventeurs ${ }^{2}$.

Bally est donc de plus en plus cité, mais il ne semble pas toujours vraiment lu. Ses textes sont trop souvent une espèce d'auberge espagnole, où chacun-e va chercher ce qui l'arrange. Il est vrai que la construction quelque peu fragmentée de son discours facilite ce type de pratique. Il n'empêche qu'ainsi des pans entiers de sa réflexion sont ignorés. Maint-e-s admirateurs et admiratrices semblent se limiter à la lecture de son petit essai, certes fort stimulant, Le langage et la vie. Bally pourrait d'ailleurs avoir encouragé une telle pratique puisqu'il présente cet opuscule comme une introduction à son œuvre fondamentale, Linguistique générale et linguistique française, dont la lecture peut donc toujours être repoussée à plus tard.

De même qu'il y a eu une vulgate saussurienne, que l'on doit en grande partie à Bally luimême, de même assiste-t-on à une sorte de vulgate ballyenne, qui se concentre sur les concepts d'énonciation, de stylistique, de modus ou de dictum, d'expression affective ou intellectuelle, de style indirect libre, de marqueurs sociaux, etc.

Il ne s'agit aucunement pour moi de nier ces dimensions, fondamentales il est vrai ${ }^{3}$. J'aimerais cependant insister sur d'autres problématiques, certes moins visibles, mais néanmoins insistantes, qui relèvent de la linguistique générique ${ }^{4}$ ou de ce qui, dans le monde anglo-saxon, est parfois désigné comme la sphère du "Language \& Gender"5. Bally développe à plusieurs reprises bien des problématiques et des argumentaires que l'on (re)découvrira des années plus tard sous la plume des plus célèbres pionnières de la linguistique générique, parmi lesquelles je me contenterai de citer Robin Lakoff, Jacqueline Lamothe, Luise F. Pusch, Dale Spender, Senta Trömel-Plötz et Marina Yaguello.

Bien que le domaine de la linguistique générique ne date donc pas d'hier, il est toujours en quête d'institutionnalisation dans un certain nombre de régions ou de pays, parmi lesquels se trouvent la Suisse romande et la France. Cet article a pour objectif de montrer l'épaisseur historique d'une problématique qui, d'une part, n'est pas linéaire et progressive et qui, d'autre part, est non seulement insuffisamment reconnue mais tout simplement mal connue sur le sol francophone européen. Il s'agit ici de rendre visibles et peut-être même pertinentes des

\footnotetext{
2 Cet article est le résultat de nombreuses discussions. Je remercie celles et ceux qui ont bien voulu y prendre part et plus particulièrement Daniel Elmiger, Jean-Daniel Gollut, Jérôme Meizoz, Mireille Noël, Stéphanie Pahud et Robert Vion, ainsi que les étudiant-e-s de l'Université de Lausanne.

${ }^{3}$ Je les ai d'ailleurs abordées de façon critique dans Durrer 1998.

${ }^{4}$ Ce terme est construit sur le modèle d'autres désignations comme linguistique textuelle, linguistique conversationnelle, linguistique pragmatique, linguistique systémique, etc.

${ }^{5}$ Cette désignation se trouve notamment sous la plume de Victoria L. Bergvall dans un numéro précédent de Linguistik Online 2, 1/99.
} 
Les femmes et le langage selon Charles Bally : "des moments de décevante inadvertance"?

interrogations et des interprétations trop souvent occultées par les conceptions et le découpage traditionnels du domaine linguistique. J'aimerais faire de cette réflexion autour de Bally une contribution à l'entreprise quasi archéologique que Françoise Collin, Evelyne Pisier et Eleni Varikas ont développée à propos de la question de la différence des sexes en philosophie et qui a récemment abouti au livre Les femmes de Platon à Derrida. Anthologie critique :

1. "L'enseignement traditionnel des œuvres [philosophiques] semble en effet l'ignorer [la question de la différence des sexes] ou ne la convoquer que rarement. On pourrait donc supposer qu'elle en est absente ou au mieux marginale et qu'elle ne concerne que des penseurs mineurs. On verra qu'il n'en est rien et qu'elle est au contraire présente chez la plupart des grands philosophes. Rares sont ceux qui n'y ont pas consacré au moins quelques passages attestant de son importance. Dans l'élaboration de cette anthologie nous n'avons pas dû, dans la plupart des cas, fouiller les bords des œuvres : il nous a suffi de les relire telles qu'elles se donnent ou sont constituées par la tradition universitaire, mais en portant sur elles un nouveau regard. Une fois adopté, ce regard fait surgir une matière si importante et si diversifiée qu'un volume exploratoire comme celui-ci ne peut prétendre l'analyser exhaustivement.

En effet, loin d'être inconnus ou périphériques, la plupart des textes que nous avons sélectionnés font partie d'œuvres répertoriées mais ils ont le plus souvent été neutralisés dans leur approche. C'est donc une nouvelle grille de lecture, absente jusqu'ici dans la tradition interprétative des textes et de leur transmission, qu'il s'agit de constituer et d'éprouver. Le premier effet de notre travail a été d'arracher la question qui nous occupe au voile d'invisibilité dont elle avait été jusqu'ici recouverte et d'en faire apparaître la fécondité et l'intérêt, avant même d'en dégager le sens et l'articulation propres à chaque œuvre. (Collin, Pisier \& Varikas $2000: 7$ )

La publication toute récente de deux autres recherches, qui procèdent à une sorte d'inventaire des lieux et des formes de problématisation du genre grammatical, témoignent d'une vague de "fouilles", qui pourraient augurer un nouveau palier dans la lente institutionnalisation de la linguistique générique 6 .

En 2001, Claudie Baudino a livré les résultats de son travail de doctorat dans un livre intitulé Politique de la langue et différence sexuelle. La politisation du genre des noms de métier. Elle y traite notamment d'un certain nombre d'auteur-e-s, linguistes ou grammairien-ne-s, préoccupé-e-s par la question, parmi lesquel-le-s une place particulière est attribuée à Ferdinand Brunot, Jacques Damourette et Edouard Pichon, Otto Jespersen, William Labov, Luce Irigaray, Georges Dumézil et Claude Lévi-Strauss. On notera qu'aucune mention n'est faite de Charles Bally.

Plus récemment encore, Claire Michard nous a donné à lire une réflexion sur Le sexe en linguistique. Sémantique ou zoologie? Les analyses du genre lexical et grammatical des années 1920 aux années $19700^{7}$ (2002). La période retenue nous intéresse au premier chef dans

\footnotetext{
6 J'ajouterai à ces deux réflexions un article très intéressant de Christine Planté sur "quelques discours sur l'E muet" (2001).

7 Claire Michard annonce un deuxième volume consacré au développement de la problématique dans les années 1970 à 1990 et notamment aux réflexions issues des mouvements féministes après 1968.
} 
la mesure où elle inclut - outre Jespersen ou Damourette et Pichon - Antoine Meillet, Marguerite Durand, Roman Jakobson, Louis Hjelmslev, André Martinet, Istvan Fodor, Jean Dubois et André Joly. Une fois de plus, on constate qu'il n'y est jamais fait allusion à Charles Bally, dont les réflexions méritent cependant, à mon sens, d'être mises en lumière et en perspective.

De façon quelque peu sommaire, je rappellerai qu'on peut regrouper les problématiques abordées par les chercheuses et les chercheurs qui travaillent dans le domaine de la linguistique générique sous quatre chapitres, dont les trois premiers ont fait l'objet, comme nous le verrons dans cet article, de réflexions, plus ou moins approfondies, de la part de Charles Bally :

a) La place du féminin et du masculin dans le système linguistique

b) Les pratiques des femmes et des hommes dans la conversation

c) La représentation des femmes et des hommes dans les discours oraux ou écrits

d) Les pratiques des femmes et des hommes dans la communication écrite

Claudie Baudino et Claire Michard, et la majorité des auteur-e-s qu'elles citent, se sont essentiellement centré-e-s sur la première question. A la différence de la plupart de ses confrères, Bally ne s'est pas contenté de réfléchir sur la seule question du genre grammatical, mais a parcouru, certes de façon lapidaire parfois, presque tout le domaine de la linguistique générique.

Signalons d'emblée que Bally n'a pas consacré un texte en particulier aux questions génériques. Il a livré ses réflexions, de façon éparse, principalement dans divers chapitres et paragraphes de deux ouvrages : Le langage et la vie ( $1^{\mathrm{e}}$ éd. 1913) d'un côté et Linguistique

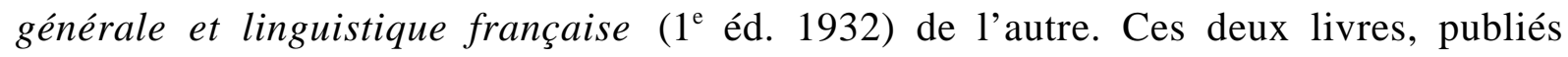
postérieurement à l'édition du Cours général de linguistique (1913) de Ferdinand de Saussure, ont fait l'objet de plusieurs moutures, au fil desquelles, il faut le noter, la réflexion générique s'est affirmée avec une vigueur croissante. Le Précis de stylistique (1905) et le Traité de stylistique française (1909), qui contiennent en germe la théorie énonciative de Bally, développée et sédimentée par la suite dans Linguistique générale et linguistique française, ne présentent en revanche aucune dimension relevant de la linguistique générique ${ }^{8}$.

\section{$1 \quad$ Genre grammatical vs genre naturel ?}

Une des questions qui a beaucoup occupé la linguistique générique occidentale dès ses débuts concerne les relations entre le genre grammatical et le sexe, également qualifié de "genre naturel". Ces dernières années, une telle question a cependant été considérée comme non

8 Par la suite je renverrai par les abréviations suivantes aux textes de Bally : TSF (Traité de stylistique française), LV (Le langage et la vie), enfin LG et LF (Linguistique générale et linguistique française). 
Les femmes et le langage selon Charles Bally : "des moments de décevante inadvertance"?

pertinente par une grande partie de la linguistique institutionnelle au nom de l'arbitraire du signe tel qu'il a été développé dans la vulgate saussurienne. Etonnamment, du moins à première vue, Bally, l'éditeur du Cours de linguistique générale de Saussure, répétons-le, interroge cette approche. Bally n'envisage pas la question des genres dans une perspective purement formelle, structurale. Dans les premières lignes d'un chapitre de son essai Le langage et la vie, Bally considère, de façon assez frontale, les valeurs ou même les connotations sémantiques de phénomènes morpho-syntaxiques comme un fait social, expression qu'il avait déjà employée dans son Traité de stylistique française mais dans une acception quelque peu différente ${ }^{9}$. Cette expression sera d'ailleurs également utilisée dans le Cours, mais là encore dans une autre perspective. Pour Bally, la langue est un fait social dans la mesure notamment où ses structures et ses choix sémantiques, morpho-syntaxiques, lexicaux, etc. sont en relation, en résonance avec une idéologie ou des croyances collectives, dimensions qui ne sont pas abordées dans le Cours :

2. Je formulerai un postulat dès le début; il dominera toutes les vues que j'exposerai ici ; au lecteur d'en mesurer la justesse.

La langue n'est pas seulement, par définition, un fait social ; c'est de toutes les institutions sociales, celle qui nous rapproche le plus des origines de la société, parce que c'est la plus instinctive, la plus traditionnelle, celle enfin dont l'emprise sur les individus est la plus forte.

On se préoccupe beaucoup aujourd'hui de découvrir dans les sociétés évoluées comme les nôtres, des traces de la mentalité primitive: superstitions, magie, symbolisme, contradictions commandées par des sentiments collectifs, etc. La langue fournirait, je crois, des indices abondants de ces survivances. On verrait, par exemple, que son évolution est toujours en retard sur celle de la pensée moyenne, si bien qu'elle impose à l'esprit le plus abstrait des formes mythiques, illogiques ou enfantines. Il faudrait de longs développements pour prouver ce point, et quelques exemples ne suffiront pas pour le mettre en lumière. On pourrait citer les genres des substantifs, qui personnifient en quelque sorte les objets, en font des personnages de contes de fées (le soleil, la lune) et attribuent souvent un seul sexe aux êtres sexués (un moineau, une hirondelle); l'araignée évoque des représentations différentes selon que son nom est du féminin, comme en français, ou du masculin, comme en russe ; le neutre fait des personnes des êtres insexués (allemand das Weib, das Kind). (Bally LV : 115) ${ }^{10}$

A travers cet extrait, il apparaît assez nettement que, dans une perspective générique, Bally n'opère pas de disctinction entre les animés des inanimés, à la différence d'un certain nombre de linguistes pour lesquels l'identification sexe et genre ne vaudrait que pour certains animés et serait totalement impertinente pour les inanimés. Pour Bally, l'assimilation est pertinente dans tous les cas : pour les animés, animaux ou humains (un moineau, une hirondelle, das Mädchen) comme pour les inanimés (le soleil, la lune) pour les concrets (la rose) comme pour les abstraits (le Vice, la Vertu).

9 "Le langage est encore un fait social au premier chef parce qu'il classe d'une manière ou d'une autre le sujet parlant. Sans doute, il le classe individuellement avant de le classer socialement; de plus, lorsqu'il le classe dans un groupe, ce groupe est une réalité mal définie, il n'a pas la rigidité d'une classe sociale au sens strict du mot; c'est un milieu [...]". (TSF I : 10)

10 Sauf mention explicite, tous les soulignements et gras sont le fait des auteur-e-s cité-e-s. 
Selon Bally, le genre grammatical n'a pas seulement une valeur distinctive et syntaxique mais il a également une valeur sémantique. Ce faisant, Bally défend un point de vue très radical, qui le place à une des extrémités du continuum des relations entre sexe naturel et genre grammatical, tel que le décrit Claire Michard:

3. Quant à la définition sémantique, elle varie entre deux pôles extrêmes : le genre n'a aucun sens ou le genre a un seul sens pour tous les substantifs. Dans ce deuxième cas, le fondement sémantique est le sexe ou un trait conceptuel abstrait. Certains auteurs adoptent une position intermédiaire : le genre signifie le sexe pour les termes désignant les humains (et dans certains cas les animaux), il ne signifie rien pour les termes désignant les non-animés, mais il peut être exploité métaphoriquement. (Michard $2002: 31$ )

La conception représentée par Bally est loin de faire l'unanimité et sera combattue avec une extrême virulence bien des années plus tard par d'autres éminents chercheurs comme Claude Lévi-Strauss ou Georges Dumézil, qui se sont exprimés au nom de l'Académie française. C'est au célèbre anthropologue que l'on doit d'ailleurs la rédaction d'un texte qui dénonce la féminisation systématique des noms de profession, titres et fonctions, dans lequel on peut lire :

4. Il convient en effet de rappeler qu'en français comme dans les autres langues indo-européennes, aucun rapport d'équivalence n'existe entre le genre grammatical et le genre naturel. (Déclaration rédigée par Claude Lévi-Strauss au nom de l'Académie française lors de la séance du 14 juin 1984 ; cité par Baudino 2001 : 370)

Il s'agit là d'une position également défendue par des linguistes contemporains de Bally, parmi lesquels on peut citer le spécialiste danois de langue française, Kristoffer Nyrop (18581931). Dans sa Grammaire historique de la langue française, ce dernier, en stigmatisant les propos de Raoul de la Garanderie, se distancie très nettement de la perspective sémantique :

5. [...] on s'est livré à des spéculations philosophiques ou plutôt à des rêveries qui n'ont rien à voir avec la science. Selon Raoul de la Grasserie, le masculin est considéré comme supérieur au féminin. "On masculinisera les objets et les êtres qui sont réputés posséder les qualités viriles et on féminisera les autres. On donnera le masculin aux mots qui semblent représenter l'activité, la précision et la limitation ; le féminin, à ceux dont le sens est vague ou très étendu." Raoul de la Grasserie voit là une concordance physiologique rappelant l'agressivité du mâle, la passivité de la femelle. (Nyrop 1936² : 354) 
Les femmes et le langage selon Charles Bally : "des moments de décevante inadvertance"?

On peut lire dans La pensée et la langue de Ferdinand Brunot (1860-1938), autre contemporain de Bally, des propos sur les genres qui s'inscrivent dans une ligne proche de celle de Nyrop ${ }^{11}$ :

6. Dans le nom de choses qui ont été formés, ce ne sont point des considérations de sexe qui ont déterminé la répartition dans un genre ou dans l'autre, mais des analogies et des raisons de forme. (Brunot $1965: 87$ )

C'est la même conception que l'on trouvera quelques années plus tard, en 1937, sous la plume conjointe de Brunot et Bruneau :

7. Le genre des noms, dans la plupart des cas, est arbitraire : il n'y a pas de raison intrinsèque pour que le soleil, le tableau soient masculins, tandis que la lune, la table, sont féminins (et, en effet, les mots allemands correspondants sont du genre opposé). (Brunot \& Bruneau 1937 : 288-289)

Une telle position, si fréquemment tenue ces dernières années ${ }^{12}$, n'aurait à l'évidence pas été partagée par Charles Bally. S'il n'y a, au départ, pas nécessairement de raison sémantique pour que le soleil soit masculin en français et féminin en allemand, il y a néanmoins, à l'arrivée, des interprétations qui ne sauraient être balayées d'un revers de main. Pour le linguiste genevois, la réception des sujets parlants ne peut être ignorée des linguistes, du moins de ceux qui se situent dans une perspective synchronique ; ces derniers ont en effet pour tâche de répertorier et de comprendre les conceptions et les interprétations des sujets parlants ; il s’agit même là du cœur de la perspective synchronique :

8. Car si la diachronie est la linguistique des linguistes, la synchronie est celle des sujets parlants ; la science doit ici se borner à décrire et à systématiser leur conception de la langue. (Bally 1940 : 203)

Selon Bally, le sujet parlant, qu'on le veuille ou non, attribue une valeur sémantique au genre morphologique. Ce phénomène lui paraît suffisamment important pour qu'il l'évoque à plusieurs reprises :

9. Le concept qui surgit en nous quand nous prononçons le mot femme est façonné, non par la réalité, mais par les associations qui relient ce mot à d'autres mots, porteurs d'idées également traditionnelles; ainsi femme a un sens plus étendu que l'allemand Weib, parce que ce dernier est limité par le synonyme Frau, limitation que ne connaît pas le français ; mais d'autre part, pour Weib la coloration affective est différente de celle de femme, parce que le mot allemand est du genre neutre ; cette étiquette, appliquée sur un être du sexe féminin, pousse l'imagination dans une toute autre direction que le féminin femme. On pourrait en dire autant de Kind (neutre), comparé à enfant, presque toujours masculin malgré la dualité (cf. des expressions comme "un enfant du sexe féminin"). (Bally LV : 123)

\footnotetext{
11 Il est intéressant cependant de trouver ici et là des observations qui atténuent quelque peu la conception générale : "A cette distribution s'attache une idée de valeur : l'encens mâle est plus apprécié que l'encens femelle. C'est dans le même sens qu'on dit : les effets sont mâles et les promesses femelles." (1922: 85) 12 Pour une présentation critique et une contextualisation de ces débats, cf. Baudino 2001.
} 
Les mots prennent une valeur non seulement du fait de leur appartenance à un système lexical mais aussi par leurs caractéristiques morphologiques. Il n'est pas indifférent qu'un mot soit féminin, masculin ou neutre. Le genre grammatical participe du sémantisme ; cette valeur sémantique des genres grammaticaux ne constitue pas une problématique anecdotique pour Bally, qui la traite à plus d'une reprise :

10. Le français s'est débarrassé du neutre, mais conserve la distinction entre le masculin et le féminin ; la répartition des substantifs entre les deux genres est des plus capricieuses : grosse surcharge pour la mémoire! Mais la fantaisie ne trouve-t-elle pas son compte à cette complication ? On a beau dire que le genre grammatical est une pure survivance sans signification : le seul fait de mettre le ou la devant un substantif lui donne une personnalité. La rose serait-elle la rose si elle changeait de genre ? Il y a du folklore dans l'opposition entre le soleil et la lune; le genre personnifie les abstraits comme des entités mâles ou femelles (le Vice, la Vertu ; cf. 139). Restes de la mentalité primitive si l'on veut, mais encore bien vivants. (Bally LG \& LF, nº02 : 364)

Où situer exactement le sens livré par le genre grammatical ? A lire Bally, l'interprétation sémantique du genre relève non pas de la parole mais de la langue :

11. La quantification du substantif fait apparaître la profonde différence qui sépare la catégorie du nombre de celle du genre grammatical ; le nombre relève, comme on le voit, de la quantification actuelle, et est du domaine de la parole (119); le genre caractérise (qualitativement) le concept virtuel et appartient, par conséquent, à la langue. (Bally LG et LF, nº115 note 1 : 139)

Le genre fait donc partie du signifié, même à l'état virtuel. Ces quelques lignes sont particulièrement importantes pour une autre raison encore : Bally y interroge le regroupement, traditionnellement opéré, au sein d'un seul chapitre de grammaire des questions de nombre et de genre, car une telle pratique aboutit à l'assimilation indue de deux types de variation en fait bien distinctes.

Ce n'est donc pas dans un contexte particulier que des connotations, des effets sont produits, mais ils sont inhérents aux substantifs :

12. Mentionnons un autre cas-limite de la caractérisation : le genre grammatical (115 n.). Il importe peu qu'il ait sa marque propre dans le substantif lui-même, p. ex. sous la forme d'un suffixe qui ne comporte qu'un genre, comme dans fr. audi-teur, audi-trice, all. Mäd-chen, ou qu'il soit déterminé seulement par la forme des actualisateurs ou des adjectifs accordés avec lui (cette table, air vif): dans les deux cas, le substantif, même isolé de tout contexte, est "pensé" avec son genre : table est féminin en tant que mot. Or, le genre présente les choses à l'imagination sous certains aspects, avec certaines particularités, si vagues soient-elles (117); le genre caractérise donc, à sa manière, les substantifs virtuels. (Bally LG et LF, $\mathrm{n}^{\circ} 139$ : 92)

Pour reprendre une terminologie que Bally utilisera dans son article de 1940 sur la valeur et la signification, la dimension sémantique du genre ne relève pas de la signification mais de la valeur. Ce n'est pas à l'occasion de telle personnification dans tel poème ou autre type de 
Les femmes et le langage selon Charles Bally : "des moments de décevante inadvertance"?

discours que le soleil devient mâle et la lune femelle, c'est en eux-mêmes que ces deux mots sont porteurs, en français, de ces dimensions sexuées, qui peuvent cependant être exacerbées dans des contextes particuliers.

Bien que Bally ne fasse pas usage de la notion de sexuisemblance, créée par ses atypiques confrères Damourette et Pichon ${ }^{13}$, il soutient un point de vue qui le rapproche beaucoup de ceux dont le travail a souvent été mis en évidence par la linguistique féministe, alors même que leur approche est souvent imprégnée de considérations patriarcales. Voici dans quels termes ces deux savants présentaient cette notion :

13. Les substantifs nominaux y sont, nous le voyons, répartis en deux groupes distincts et bien définis. Les uns (chien, évêque, sang, dragon, foin, couvent) sont masculins, c'est-à-dire expriment des substances de sexe masculin ou comparées par la langue à des êtres mâles; les autres (vie, filles, femmes) expriment des substances du sexe féminin ou comparées par la langue à des êtres femelles. Le répartitoire qui établit ces deux groupes a reçu de nous le nom de répartitoire de sexuisemblance. (Damourette \& Pichon 1911-1927 : 347)

Un peu avant Bally, Damourette et Pichon, dont les travaux semblent avoir été connus du linguiste genevois, avaient donc considéré que le genre, masculin ou féminin, fait complètement partie du signifié. Ils vont même jusqu'à préciser qu'il s'agit d'un sème à part entière dénommé sémième dans leur terminologie bien spécifique :

14. Dans le substantif nominal, la sexuisemblance est une propriété fondamentale inséparable du concept envisagé, invariable, indépendante de tout élément extérieur, faisant partie du sémantisme même du vocable. C'est le sémième de chaque substantif nominal qui est masculin ou féminin. (Damourette \& Pichon 1911-1927 : 357)

A l'instar de Bally, Damourette et Pichon revendiquent une approche fondamentalement synchronique, qu'ils exposent en recourant de façon très insistante à la notion d'actualité :

15. Au surplus, à supposer même que la loi d'irradiation, l'analogie ou tout autre mécanisme étymologique permissent de rendre compte, dans chaque cas particulier, des causes qui ont fait classer tel substantif comme masculin, tel autre comme féminin, ce procédé simplement historique, serait encore absolument impuissant à nous rendre compte de la nature actuelle et du rôle actuel du répartitoire de sexuisemblance dans le français actuel. (Damourette \& Pichon 1911$1927: 360)$

Damourette et Pichon ajoutent plus loin que "le problème essentiel, le problème sémantique est de savoir ce qu'est pour le psychisme du locuteur français la sexuisemblance" (1911$1927: 361)$.

\footnotetext{
13 Pour une présentation de ces deux savants tout à fait hors norme, lire l'article d'Hélène Huot 1991 dans le très intéressant ouvrage qu'elle a édité sur La grammaire française entre comparatisme et structuralisme 1870-1960. Ce livre contient notamment des contributions de Jean-Claude Chevalier sur Ferdinand Brunot et de René Amacker sur Charles Bally.
} 
Tout cela ne signifie pas pour autant que le choix de l'appartenance générique ait été motivé de façon simple et immédiate par une représentation sexuée de la réalité extra-linguistique inanimée. Dans la perspective de Damourette et Pichon, comme dans celle de Bally cidessous, cela veut dire que le genre grammatical d'un nom est en relation avec l'imaginaire sexué d'une collectivité, qu'il contribue largement à construire :

16. [...] nous apercevons souvent la réalité à travers le prisme de la langue ; c'est là un des aspects de la psychologie linguistique qu'on néglige trop dans l'étude des rapports entre le langage et la pensée. L'exemple le plus caractéristique de ce phénomène nous est offert par la manière dont nous classons les objets et les procès de la réalité. Qu'il s'agisse de catégories générales ou de distinctions particulières, ce triage est subjectif, puisqu'il diffère en tout ou partie d'un idiome à l'autre. C'est ainsi qu'en français les êtres et les choses doivent être masculins ou féminins, et quoiqu'on le conteste souvent, cette estampille sexuelle les présente à l'esprit sous un aspect où la fantaisie trouve son compte; pensez à des couples tels que pied et main, fauteuil et chaise, soleil et lune, sans compter ceux que les psychanalystes interprètent de façon plus précise : bouchon et bouteille, tenon et mortaise, pène et gâche, etc.). On sait que beaucoup d'animaux, grâce à la langue, ne peuvent être que mâles ou femelles (le requin et la baleine, le rat et la souris, etc.); on a peine à se figurer que l'hirondelle serait le même oiseau si elle changeait de genre; et le folklore imagine des rapports sentimentaux entre le pinson et la fauvette. (Bally 1940 : 197)

Pour Bally, il est donc indéniable que les genres produisent ces effets, qu'il ne se contente pas de constater, mais qu'il peut être amené à dénoncer. En effet, bien des fonctionnements linguistiques, parmi lesquels se trouvent les genres, seraient des reflets non pas de la réalité, mais d'une représentation du réel, qui serait en général dépassée : "[l'] évolution [de la langue] est toujours en retard sur celle de la pensée" (LV : 115). Bally ne se limite pas à poser l'existence d'une interprétation "sexuisemblante", qui ferait de la langue un simple reflet (LV : 115) mais il pousse plus loin le rapport entre la langue et la réalité, en faisant de certaines caractéristiques linguistiques des freins à l'évolution dans un premier temps cognitive : "[la langue] impose à l'esprit le plus abstrait des formes mythiques, illogiques ou enfantines". Les genres grammaticaux seraient donc également vecteurs d'effets pragmatiques. Bally tient un point de vue plus critique que Damourette et Pichon :

17. A vrai dire, peu importe de savoir si la forme d'où le français a tiré ce vocable [la mer] était mare ou, comme l'a supposé Darmsteter, maris. L'essentiel, c'est qu'il est passé au féminin dans notre langue, alors qu'il demeurait au masculin dans d'autres langues romanes. Et il semble difficile d'expliquer cette modification autrement que par des besoins métaphoriques conformes à l'esprit national, la mer ayant été conçue par nos ancêtres, de même que par nous, comme quelque chose de féminin. La mer est d'aspect changeant comme une femme, journalière, d'humeur mobile comme une jolie capricieuse, attirante et dangereuse comme une beauté perfide. (Damourette \& Pichon 1911-1927 : 371)

Damourette et Pichon se contentent de relayer des associations sans évoquer leur caractère socialement construit ni stigmatiser leur axiologisme. Je ne saurais cependant dire de façon aussi radicale que Claire Michard que "cette analyse est exécrable quand on est de sexe 
Les femmes et le langage selon Charles Bally : "des moments de décevante inadvertance"?

femelle parce qu'elle se complaît dans l'idéologie naturaliste sexiste sans la traiter comme telle" (2002 : 52). Il n'y a, il est vrai, pas de dénonciation du sexisme, pas même reconnaissance du statut sexiste de certaines caractéristiques, mais il ne me semble pas qu'il faille voir dans les propos de Damourette et Pichon une idéologie exclusivement naturaliste. Pour reprendre les termes de Claire Michard, on ne saurait considérer que les analyses de Damourette et Pichon manifestent de façon simple et directe "le syncrétisme de l'idéologie naturaliste, entre sexe biologique (femelle) propre à toutes les espèces sexuées et genre sociopsychologique (féminin) propre aux humains" (Michard 2002: 83). Pour ces deux grammairiens, ce qui est à la base de la sexuisemblance, ce n'est pas la nature des référents mais l'impression qu'ils provoquent sur une collectivité particulière, étant donné sa conception du monde :

18. [...] la sexuisemblance n'étant pas une notion scientifique, mais intuitive et poétique, n'a pas pour base la notion scientifique de sexe, dans toute son extension biologique, mais bien uniquement les réactions impressives provoquées par l'un ou l'autre sexe. Ce sont donc avant tout les sexes de l'espèce humaine qui sont le point de départ de la métaphore sexuisemblantielle. (Damourette \& Pichon 1911-1927 : 419)

Il est toutefois clair que Damourette et Pichon ne discutent pas et encore moins contestent ces impressions.

Bally ne développe pas de façon aussi détaillée que ses confrères les effets de la dimension générique d'un substantif. Il reste très vague sur les valeurs sémantiques du masculin et du féminin. Bally associe bien les caractéristiques féminines "naturelles" et le sème "féminin", mais, à la différence de Damourette et Pichon, qui détaillent ce sème et qui l'identifient avec la petitesse, la finesse, la sexualité, l'imprécision, la passivité, la sous-humanité, l'animalité, etc., Bally se contente d'évoquer des "rapports sentimentaux entre le pinson et la fauvette", sans attribuer un rôle bien défini à l'une ou à l'autre instance et sans spécifier la nature exacte des rapports sentimentaux.

Bally assume une perspective malgré tout plus critique que Damourette et Pichon - pour lesquels la langue française possède un véritable "génie" jamais pris en défaut - car il dénonce certains aspects par trop conservateurs de la langue ; il s'oppose ainsi à d'autres points de vue qui voudraient que les changements sociaux induisent presque nécessairement des changements linguistiques, fût-ce avec un certain décalage. Si la langue peut constituer un frein à l'évolution cognitive (cf. citation $\mathrm{n}^{\circ} 1$ supra), il lui arrive également de retarder certaines évolutions sociales et politiques :

19. On admet, le plus souvent sans preuves à l'appui, que l'évolution de la langue et celle de la société sont parallèles et synchroniques, que tout changement dans la vie sociale et politique du groupe doit avoir, de manière automatique, immédiate et unilatérale, son contre-coup dans la structure de la langue. Mais d'abord, y a-t-il nécessairement parallélisme ? On sait combien une langue est ouverte aux influences du dehors [...]. Ensuite - et voici qui est paradoxal, - la langue est en même 
temps la plus traditionnelle de toutes les institutions sociales, celle dont l'évolution est la plus lente. [...] La conséquence est qu'elle peut très bien refléter une mentalité collective qui s'est profondément modifiée au cours du temps. [...] En conséquence, vouloir trouver une correspondance constante entre langue et culture, surtout si l'on base l'argumentation sur la langue littéraire et le style des grands écrivains, c'est là une entreprise séduisante sans doute, mais qui réserve, je le crains, bien des désillusions. (Bally LG et LF : 14)

Pour Bally, à l'instar d'autres grammairiens, les formes ne sont pas coupées du sens. Toutefois, son analyse se distingue par deux caractéristiques. Premièrement, pour Bally, la langue est très conservatrice ; elle n'accompagne pas suffisamment les changements sociaux, qu'elle tend à entraver et trop souvent continue d'abriter des conceptions dépassées. Bally a développé un tel point de vue tant sur les questions génériques que sur les questions orthographiques $^{14}$. Deuxièmement, à la différence de Brunot ${ }^{15}$ par exemple, Bally refuse le point de vue diachronique et ne veut pas entrer en débat sur l'origine et la légitimité de l'investissement sémantique des genres grammaticaux. Il se contente d'en constater l'existence. La linguistique comme la grammaire se doit donc de rendre explicites ces sens latents, quelle que soit leur pertinence historique. Il revendique, sur ce plan, une visée sémantique globale comparable à celle de Ferdinand Brunot, telle qu'elle apparaît dans $L a$ pensée et la langue (1922), même si Bally peut s'opposer à son collègue sur un certain nombre de questions parmi lesquelles se trouve l'interprétation des genres grammaticaux, comme on l'a vu plus haut (cf. citation $n^{\circ} 4$ et $\left.n^{\circ} 5\right)$ :

20. [...] le souci de rattacher systématiquement le signifiant au signifié deviendra, il faut l'espérer le souci de tous les grammairiens; et puisque l'on constate qu'en plein XXe siècle la plupart des manuels courants parlent de temps, de modes, de pronoms, etc., sans y voir autre chose que des formes à prendre telles quelles, ou des prétextes à formuler des règles orthographiques, l'avertissement de M. Brunot est salutaire; on y regardera à deux fois avant de décrire un fait de grammaire sans essayer de dire quelle forme de pensée peut s'y couler. (Bally 1922 : 137)

Bally, à l'instar de Brunot, appelle les grammairiens à sémantiser les formes. Ailleurs toutefois, Bally met en garde contre les voies de cette sémantisation, qui varie par trop selon que l'on porte son attention à sa langue maternelle ou à des langues plus ou moins exotiques. Trop souvent, les préjugés sexuels, géographiques, etc. interfèrent, de façon non consciente, avec une description donnée comme étant objective. Il est donc urgent de procéder à des décentrements, par rapport à son inscription ethnologique comme à son déterminisme sexuel. Il s'agit d'opérer sur sa langue le même type d'approche détachée et critique que celui que l'on pratique sur des idiomes éloignés :

21. Il paraitt aussi qu'un peuple sauvage a un nom différent pour désigner chaque pied du cheval ; mais qu'on se rappelle les noms donnés par les chasseurs allemands aux oreilles et aux pattes des

14 Cf. son ouvrage "révolutionnaire" La Crise du français. Pour une présentation de cette problématique chez Bally, cf. Durrer 1998 chapitre 6 surtout.

15 Pour une approche comparative détaillée, voir l'article de Jean-Claude Chevalier in Huot 1991. 
Les femmes et le langage selon Charles Bally : "des moments de décevante inadvertance"?

animaux (p. 49). Un voyageur anglais reproche à une langue de non civilisés d'employer le même mot pour aimer quand il s'agit d'un ami ou d'une chose comestible; cet Anglais voit la chose à travers sa propre langue, qui distingue to love et to like; mais alors les Français sont des sauvages, puisqu'ils disent indifféremment aimer une femme et aimer le pot-au-feu!

Dans certaines langues non civilisées, les femmes sont mises, pour le genre, au rang des choses inanimées. Voilà qui est monstrueux; mais il y a mieux encore. Nous connaissons une autre langue où un verbe transitif, tel que tuer, aimer, a son régime direct au génitif s'il s'agit d'êtres animés, et à l'accusatif pour les objets inertes. On tue un homme, un bœuf, un cheval, au génitif ; on détruit un mur, on lance une flèche, de la boue, à l'accusatif. Le plus curieux de l'affaire, c'est qu'une femme est classée parmi les choses inanimées : on ne peut pas la tuer comme un bœuf, au génitif ; elle n'a droit qu'à l'accusatif, comme la boue ; pour bénéficier du génitif, il faut qu'il y en ait plusieurs : la quantité compense la qualité. Et si un animal, comme le chien, par exemple, a l'idée saugrenue d'adopter le genre féminin, immédiatement il tombe au rang de la femme, il est condamné à l'accusatif. Voilà, direz-vous, une langue bien primitive ! Pourtant c'est la langue de Tourguenieff et de Tolstoï. Si les féministes de Moscou croient au progrès linguistique, ils devront réclamer sans retard le droit au génitif, pour les femmes russes. Je n'ai pas altéré la réalité de ce point de grammaire; je l'ai seulement exposé comme on l'aurait fait s'il s'agissait d'une langue polynésienne : la leçon est bonne à retenir. (Bally LV : 51)

Bally nous amène ici à questionner ce que nous désignerions aujourd'hui par les termes d'ethnocentrisme et d'androcentrisme, voire de gender blindness. Il nous oblige à réfléchir sur les présupposés idéologiques qui guident les descriptions prétendument objectives que les linguistes occidentaux livrent d'un certain nombre de phénomènes. Au vu de ce seul passage, on pourrait cependant s'interroger sur la position de Bally. Ce dernier condamne-t-il les interprétations de phénomènes syntaxiques qui sont pratiquées, essentiellement ou exclusivement, sur des langues étrangères voire primitives ? Bally en appelle-t-il à une ethnologie de proximité, à une investigation à l'identique des langues familières comme des langues étrangères ? Quelle est au fait la leçon de Bally ? Quel point de vue défend-il ? Bally adhère-t-il à l'accusation de sexisme implicite ou adopte-t-il un point de vue ironique, qui laisse entendre non pas le contraire mais l'impertinence de ce type de problématique ? Pris isolément, ce fragment pourrait nous faire opter pour une interprétation ironique ; toutefois, les autres réflexions nous incitent à la dépasser.

La nécessaire sémantisation des formes, demandée par Bally, semble ne pas être restée lettre morte. En effet, l'approche sémantique s'est largement développée ces dernières années, même si on peut avoir le sentiment qu'elle n'a pas profondément touché le domaine des genres grammaticaux, dont l'interprétation sémantique a souvent été laissée aux polémiques journalistiques et au militantisme féministe. De façon à la fois discrète et insistante cependant, un certain nombre de grammairiens et de linguistes, bénéficiant souvent d'une forte légitimité sur le plan de la reconnaissance institutionnelle, ont continué de défendre une approche sémantico-pragmatique des genres grammaticaux, sans, il est vrai, en faire une question phare de leur réflexion. Parmi ceux-ci je mentionnerai, à titre d'exemple, Henri Bonnard qui, au début des années 70, rédige un article assez développé sur le "genre" pour le Grand Larousse 
de la langue française en sept volumes, dans lequel on peut notamment lire les propos suivants :

22. On peut considérer que le genre, à côté de sa fonction syntaxique, assume une fonction sémantique : l'indication du sexe des êtres vivants et, peut-être, à défaut de sexe, dans l'objet désigné, l'indication de certaines qualités subtiles, essentielles ou conférées [...]. (Bonnard 1972 : 2202)

Pour Bonnard, la référence essentielle en la matière, probablement à juste titre, est l'analyse de Damourette et Pichon. Bally, qui a subi une éclipse importante rappelons-le ${ }^{16}$, n'est à aucun moment cité :

23. Mais la compétence de psychiatre du docteur Pichon l'a poussé à analyser plus hardiment que tous ses devanciers les valeurs affectives du genre, dont l'existence ne peut être mise en doute. Il est certain que le genre féminin donne à la souris (masculine en latin) une "gentillesse trottinante" que nous apprécions chez Mickey Mouse, bien étrangère aux rats des fables de La Fontaine (dont le nom vient de rate). (Bonnard 1972 : 2207)

On peut toutefois s'étonner de l'exemple choisi par Bonnard car Mickey Mouse est un personnage, américain, de sexe masculin... Il n'en demeure pas moins que Bonnard, à l'instar d'un certain nombre de ses confrères, donne la sémantisation du genre grammatical, en corrélation avec le sexe, comme une pratique connotative inévitable :

24. Les facteurs formels y jouent un grand rôle, les facteurs de sens un plus grand. Le sexe est de ces derniers et peut, à ce titre, déterminer le genre (cf. les gens, la jument). Quant à la "sexuisemblance" (intuitivement détectable), elle n'est jamais qu'un effet, une connotation de genre. (Bonnard 1972: 2211)

Plus près de nous, on évoquera La grammaire d'aujourd'hui, ouvrage rédigé par trois linguistes qui ont pignon sur rue, Michel Arrivé, Françoise Gadet et Michel Galmiche. Dans leur long article consacré au "genre", on peut lire le constat suivant :

25. Toutefois l'opposition des traits non marqué / marqué n'efface pas l'opposition masculin / féminin, comme le pensent certains linguistes insuffisamment attentifs à l'investissement sémantique de la catégorie. (Arrivé, Gadet \& Galmiche 1986 : 282)

Une fois de plus, il apparaît que l'on ne saurait faire l'économie, dans une perspective synchronique, d'une valeur sémantique du genre grammatical. Arrivé, Gadet et Galmiche ne se contentent pas de cette allusion mais la développent longuement en faisant, à l'instar de Bonnard, référence à Damourette et Pichon :

16 Voir sur cette question Durrer 1998. 
Les femmes et le langage selon Charles Bally : "des moments de décevante inadvertance"?

26. Dépourvu de toute relation avec un référent sexuel inexistant, le genre des noms animés n'en fonctionne pas moins comme catégorie sémantique, de façon métaphorique, au niveau de la connotation. Comme l'ont remarqué Damourette et Pichon, le genre (qu'ils baptisent joliment sexuisemblance) est un sexe fictif attribué aux objets non animés. De ce point de vue, il est possible non de spéculer sur les raisons - inaccessibles - qui ont originellement (quand ?) conféré tel genre à tel nom, mais de repérer les relations qu'entretient le signifié du nom avec tel genre grammatical : la terre, passive et nourricière, la mer, de surcroît homophone de la mère, sont l'une et l'autre féminine, la seconde en dépit de son étymologie : son étymon latin mare est neutre. (Arrivé, Gadet \& Galmiche 1986 : 285)

La question de la valeur sémantique des genres grammaticaux est même abordée dans une grammaire pourtant fort succinte, La Grammaire Larousse du français contemporain que l'on doit à d'autres linguistes chevronnés du paysage universitaire français, Jean-Claude Chevalier, Claire Blanche-Benveniste, Jean Peytard et à nouveau Michel Arrivé. De manière rapide mais néanmoins claire, les auteur-e-s observent que "du point de vue du sens, le genre constitue, selon la pittoresque expression des grammairiens Damourette et Pichon, un sexe fictif" (Chevalier, Blanche-Benveniste, Arrivé \& Peytard 1974 : 164).

Si les linguistes qui défendent une approche sémantico-pragmatique des genres grammaticaux en français sont loin d'être rares, on ne saurait évidemment parler de consensus. Comme du temps de Bally, une perspective strictement morphologique existe également, on la trouve notamment soutenue par les auteurs de la Grammaire méthodique du français :

27. Les noms dénotant des référents non animés ont un genre arbitraire, masculin (le sable) ou féminin (la table). Souvent déterminé par l'étymon, parfois conditionné par des facteurs culturels (le soleil et la lune sont respectivement féminin et masculin en allemand : die Sonne, der Mond), le genre n'en reste pas moins irréductible à des oppositions sémantiques généralisables [...]. (Riegel, Pellat \& Rioul 1994 : 172)

Parmi les plus virulents opposants à une approche sémantique des genres, outre Georges Dumézil et Claude Lévi-Strauss mentionnés tout au début de cette réflexion, j’évoquerai encore Henri Morier, à qui l'on doit un article férocement polémique, paru en 1994, dans lequel il dénonce "le désir d'une sexuisemblance généralisée". Cet article est particulièrement dérangeant dans la mesure où il tend à décrire l'option sémantique de Thérèse Moreau (1991) comme totalement incongrue et typiquement issue d'une personne ignorante en linguistique. Or, comme l'ont montré les réflexions précédentes et ainsi que le rappelle Claire Michard (2002), la tendance qui consiste à clairement attribuer une valeur sémantique sexuée au genre grammatical est le fait de nombreux linguistes, parmi lesquels on peut citer, outre Bally, Bopp, Grimm, Paul, de la Grasserie, Vendryes, etc., sans compter les contemporains.

Dans l'ensemble cependant, les linguistes sont plutôt restés timides ces vingt dernières années sur cette question. Tout se passe comme si, à partir de la prise de position de ces deux illustres académiciens que sont Georges Dumézil et Claude Lévi-Strauss (cf. citation n²), le débat avait été durablement cassé : 
28. L'arsenal déployé par l'Académie a donc bien pris dans cette controverse un caractère incontournable. Mais, si quelques critiques se sont fait entendre après la publication de la déclaration, dont celle de Nina Catach, le texte de Dumézil n'a pas eu à essuyer l'ombre d'une réfutation; et, quelques années plus tard, les analyses des deux linguistes [Nina Catach et AnneMarie Houdebine] laissent voir en dépit de leur opposition explicite à la thèse des académiciens, la même gêne à critiquer de front un article signé par le savant. (Baudino $2001: 260-261$ )

Un nombre important de partisan-e-s d'une approche sémantique n'ont pas osé ou jugé souhaitable de prendre la plume pour défendre une position contestée par deux sommités internationales.

Il apparaît donc clairement que la question du rapport entre genre naturel ou sexe et genre grammatical n'était pas impertinente à une certaine époque. Elle n'était pas le fait d'une linguistique marginale et a fait couler beaucoup d'encre. Le tabou institutionnel semble s'être instauré ces dernières années, relégué dans les marges de la réflexion linguistique, dans le discours journalistique ou politique.

On notera enfin qu'une ligne de partage sépare tendanciellement les linguistes s'inscrivant dans une perspective diachronique, qui refusent le point de vue sémantique, et les linguistes synchronicien-ne-s qui affirment l'importance d'une telle dimension pour les sujets parlants. De même, il semble que les généralistes sont plus réticents au point de vue sémantique que les spécialistes du français.

Souvent en prise avec son époque et parfois en avance sur elle, Bally a participé aux débats contemporains sur les rapports entre les sexes et le langage. Il a toutefois indiqué d'autres pistes de réflexions, moins largement fréquentées, qu'il s'agit maintenant de présenter.

\section{Dériver le féminin du masculin : une habitude discutable}

Dans le cadre de considérations qui relèveraient aujourd'hui d'une linguistique générique, Bally élargit la réflexion au niveau du métadiscours. Il procède alors à une rapide analyse du discours grammairien, dont il interroge le sous-bassement idéologique :

29. L'habitude qu'ont la plupart de nos langues de dériver les noms féminins du masculin (poétesse d'après poète, all. Arbeiterin formé sur Arbeiter, etc.) n'est pas sans répercussion sur la façon dont l'esprit se représente les rapports entre sexes. (Bally LV : 115)

Bien que Bally désigne ici la question en terme de langue, il s'agit en fait d'un raccourci qui renvoie au méta-discours grammairien (cf. infra $\mathrm{n}^{\circ} 31$ ). Par ces quelques lignes lapidaires extraites du Langage et la vie, Bally engage à réfléchir sur les pratiques descriptives qui ont cours en grammaire, et qui présentent le féminin comme un dérivé du masculin. Le rapport de dérivation masculin-féminin ne constituerait pas un fait établi, ni une nécessité descriptive, 
Les femmes et le langage selon Charles Bally : "des moments de décevante inadvertance"?

mais une habitude socio-grammaticale. Nous aurions là une simple pratique sociale, ou socioprofessionnelle qui ne tire pas sa légitimité des faits eux-mêmes, mais d'usages répétés et convenus. Une telle remarque, même si elle n'est pas développée, montre que le jugement sans appel de Claire Michard sur son corpus d'auteur-e-s ne saurait s'appliquer sans autre à Bally :

30. On ne trouve jamais l'ombre d'une réflexion sociologique concernant les sexes, ne parlons même pas d'une analyse, bien qu'il y ait eu de forts mouvements féministes au début du siècle, en particulier pour obtenir le droit de vote. (Michard $2002: 145$ )

De façon claire, Bally interroge les tenants et les aboutissants d'une telle coutume grammaticale. Ce faisant, le linguiste genevois défend une position bien plus critique que Damourette et Pichon qui dans une rapide discussion de cette habitude concluaient à un "inconvénient égal de partir d'une des phases du répartitoire de sexuisemblance ou de l'autre" (1911-1927 : 290). Ces derniers, tout en reconnaissant le "caractère arbitraire" de la méthode, la pratiquent. Il y a donc bien, ici et là, quelques exceptions au jugement de Claire Michard, qui méritent d'être reconnues.

Dans son œuvre maîtresse, Linguistique générale et linguistique française, Bally revient sur la question de la dérivation, dont il propose une analyse détaillée, qui explique pourquoi il serait plus judicieux, dans le fond, de dériver le masculin du féminin, donc d'inverser la tradition :

31. En français, une vaste classe d'adjectifs à finale consonantique ont une forme identique au masculin et au féminin : rouge, jaune, faible, calme, etc. Mais beaucoup d'autres adjectifs sont caractérisés au masculin par l'absence de consonnes finale du féminin; ainsi la comparaison de eau tiède : bain tiède et de eau froide: bain froid $(d)$ nous autorise à penser que les masculins froid $(d), \operatorname{ver}(t), \operatorname{court}(t), d o u(x)$, etc., etc., ont une finale zéro. On peut en dire autant d'un certain nombre de substantifs qui présentent des variations analogues à celles des adjectifs : marchande : marchand, lionne : lion, louve : loup, etc. [...] Cette hypothèse semble confirmée par le fait que les dérivés de l'adjectif sont formés sur la base du féminin pris comme radical : grand(de) - grandeur, gross(e) - grossesse, bavard(e) - bavardage, blanch(e) - blanchir, vieill(e) - vieillir, etc. Les adverbes en -ment sont particulièrement instructifs : nouvelle-ment, heureuse-ment, etc., car ici, il y a coïncidence fortuite entre un sentiment qui est en train de se créer et l'origine du type ; on sait que le radical de ces adverbes a été autrefois un adjectif féminin (clairement = clara mente). On peut rappeler encore que la liaison de l'adjectif masculin amène souvent une forme identique au féminin : bo-n' ouvrage, heureux z époux, premie-r' ordre, etc. (LG et LF, $\mathrm{n}^{\circ} 250: 161-162$ )

De tels propos mettent en évidence le caractère particulier, pour ne pas dire idéologique, des nombreuses descriptions grammaticales qui présentent unilatéralement le féminin comme étant dérivé du masculin, donné comme la forme de base. Bally remet fortement en cause une telle perspective et va même jusqu'à l'inverser dans un nombre non négligeable de cas.

La position développée par Bally est très proche de celle qui sera soutenue bien des années plus tard, en 1978, par Marina Yaguello dans Les femmes et le langage, texte fondateur pour 
la linguistique générique en France. Yaguello commence par poser la question en se situant dans une perspective heuristique :

32. De même sur le plan de la description interne, le genre masculin est généralement considéré comme primaire et non marqué, par rapport au féminin dérivé (donc marqué). Un exemple tiré du français peut servir à montrer l'importance théorique de cette question. Faisons jouer le critère d'économie de formulation pour la description du genre en français. [...] Nul ne met en doute que le féminin soit dérivé du masculin puisque dans la majorité des cas, pour former le féminin, on se contente d'ajouter une "marque", un -e. Mais, en langue parlée, c'est-à-dire phonétiquement, la règle inverse serait plus économique. La forme du féminin étant la forme de base, on forme le masculin en retranchant la consonne finale (celle qui précède le -e muet), quelle qu'elle soit. (Yaguello $1978: 23$ )

Si Marina Yaguello justifie son inversion de la présentation traditionnelle en termes d'économie descriptive elle recourt également, faisant feu de tout bois, à l'argument historiciste :

33. Contrairement à ce qu'on pense généralement, il arrive que le masculin soit formé sur le féminin, encore que les cas n'en soient pas nombreux. Concubin vient de concubine, machin de machine, puceau a été créé par Lafontaine sur pucelle, de même que pondeur, dérivation facétieuse de pondeuse. (Yaguello $1978: 124$ )

Mais, la réflexion initiée par Bally n'a guère eu d'effets, puisque, de nos jours encore, on continue d'enseigner que le féminin dérive du masculin, sans trouver nécessaire d'interroger la légitimité linguistique d'un tel modèle descriptif, ni de réfléchir sur ses éventuelles implications idéologiques. J'en donnerai pour preuve une grammaire récente, à bien des égards novatrice, mais qui, sur ce point, relaie la description traditionnelle et passe comme chat sur braise sur son caractère critiquable :

34. Selon la tradition on forme le féminin des adjectifs en ajoutant un -e muet à la forme du masculin (grande, petite, royale, jolie ). (Riegel, Pellat \& Rioul 1994 : 358-359)

On remarquera l'expression "selon la tradition", laquelle laisse entendre que d'autres alternatives descriptives existent, qui ne seront toutefois pas mentionnées. Cette grammaire, comme tant d'autres, continue de véhiculer le préjugé selon lequel il faut ou plutôt on peut "mettre accidentellement" au féminin ce qui "est essentiellement" au masculin.

Il est à noter qu'aucun des auteurs du corpus de Claire Michard ne développe une telle réflexion :

35. Pour tous les auteurs, excepté Jespersen qui n'aborde pas la question, le genre féminin est un dérivé morphologique, soit du genre animé, dans la perspective historique de l'origine du genre dans les langues indo-européennes, soit du genre masculin dans la perspective synchronique qui 
Les femmes et le langage selon Charles Bally : "des moments de décevante inadvertance"?

domine à l'heure actuelle. Le genre féminin a donc un statut second du point de vue morphologique dans les deux perspectives. (Michard 2002 : 133-134)

Cela souligne l'originalité de Charles Bally, qui a donc été un des rares linguistes du début du siècle à s'interroger sur ce que l'on appelle aujourd'hui le déterminisme androcentrique de certaines descriptions linguistiques ou grammaticales. Très récemment, Denis Apothéloz, dans une réflexion sur La construction du lexique français (2002) attire l'attention sur la pertinence du modèle de Leonard Bloomfield (1887-1949) tel qu'il est exposé dans Language (1933) dans la mesure où celui-ci, à l'instar du modèle de Bally, remet en question l'approche traditionnelle de la consonne féminine additionnelle latente et la remplace par "l'hypothèse d'un morphème [masculin] soustractif" (2002 : 38). Un tel changement aurait pour conséquence importante de considérer que c'est "le genre masculin qui fait l'objet d'une marque morphologique. Cette marque consisterait alors en l'absence de la consonne finale" (2002 : 38). Et Denis Apothéloz conclut :

36. On notera que cette hypothèse fait du masculin la forme marquée (même s'il s'agit d'une marque soustractive) et du féminin la forme non marquée (celle qui ne comporte pas d'information de genre, si ce n'est par contraste avec la forme masculine). (Apothéloz $2002: 38$ )

De telles considérations morphologiques iraient à l'encontre des considérations sémantiques traditionnelles, dans la mesure où sur ce plan on tend à considérer que le genre masculin est non marqué et que le genre féminin est marqué (cf. Michard 2002). S'inscrivant dans une perspective purement morphologique, Apothéloz via Bloomfield ne s'interroge malheureusement pas sur la nature - éventuellement idéologique - des raisons qui ont présidé à la construction et à la résistance du modèle dit "additionnel"17.

\section{$3 \quad$ La féminisation : une violence pour le bon sens ?}

Outre la question du sens des genres grammaticaux, des choix descriptifs pour le moins discutables de l'alternance morphologique entre le féminin et le masculin, Bally aborde un autre domaine phare de la linguistique générique, qui attise régulièrement les passions dans le grand public, à savoir la féminisation d'un certain nombre de noms et d'adjectifs. Dans Linguistique générale et linguistique française, Bally évoque en premier lieu la question de la féminisation des adjectifs.

37. Dans plusieurs cas, le français recule devant l'emploi du féminin: on hésite à dire hagarde, encline, châtaine, etc. La marche à l'invariabilité est favorisée par certaines circonstances, p. ex. l'emploi régulier de l'adjectif au masculin quand il est caractérisé : "étoffe vert pâle, vert bouteille", et celui des substantifs en fonction d'adjectifs : "style gendarme, manières ogre", etc. (Bally LG et LF, $n^{\circ} 411: 255$ )

\footnotetext{
17 Edwige Khaznadar propose de renoncer aux modèles de la dérivation (additionnelle ou soustractive) au profit
} d'un modèle de l'alternance. 
La première évocation s'effectue dans une tonalité plutôt modérée. Bally traite la question en termes d'hésitation essentiellement et ne prend pas en compte ici les effets de cette timidité morphologique très ciblée. Il revient toutefois sur la question de la féminisation à travers les noms, et le ton se fait alors plus virulent :

38. Le français répugne à la formation de féminins par suffixes : doctoresse est admis (mais Mme X. est docteur ès lettres, est porteur d'un diplôme de docteur) ; cochère a eu une existence éphémère, chéfesse paraît dur ; on préfère, malgré la violence faite au bon sens, des expressions telles que : "Mme X., artiste peintre, sculpteur, décorateur; Marie-Thérèse, successeur de Charles VI ; Mme X., officier d'Académie". De là des inconséquences curieuses : "Mme L. est une romancière magnifiquement romanesque et un conteur infiniment souple. - Mme X. est appelée à faire sa déposition; le témoin affirme qu'il n'a vu l'inculpé qu'une seule fois". (Bally LG et LF, n 503 : 306)

L'hésitation devient répugnance. Cette répugnance ne semble toutefois pas être le fait de Bally, puisqu'il qualifie la pratique du masculin générique de "violence faite au bon sens". Non seulement Bally dénonce l'inadéquation inacceptable entre le genre grammatical et le sexe, mais il stigmatise les incohérences discursives et textuelles que cela entraîne. On notera que Bally ne mentionne pas le fait que les lacunes ne touchent pas indifféremment toutes les activités et professions, à la différence d'autres analyses qui mettront en évidence que ce sont surtout les professions de prestige qui souffrent d'un manque de désignations féminines.

La réflexion sur la féminisation est largement débattue du temps de Bally. Brunot l'aborde également, sous un angle purement taxinomique et non pas discursif ou textuel. Le titre met cependant clairement en évidence l'étroite imbrication du politique et du linguistique :

\section{Le développement du féminisme et les formes grammaticales.}

Il y a encore quelques fonctions essentiellement propres à chaque sexe : nourrice; marin ; chambrière; scaphandrier; soldat; cf.: rosière. Un auteur moderne a écrit une nouvelle plaisante : Le rosier de Madame Husson.

D'autres fonctions avaient apparu longtemps comme réservées aux hommes : docteur, sculpteur, ingénieur. Pour diverses causes, les portes de ces professions se sont ouvertes ou bien s'ouvrent aux femmes. Un ministre a eu pour sous-chef de cabinet sa propre fille; il y a des femmes au barreau, d'autres qui exercent la médecine, etc. La question s'est donc posée pour la langue. Quelle forme allaient prendre les titres jusque-là réservés ? Se féminiseraient-ils et comment ? Pour un certain nombre de mots, la question s'est résolue sans peine : avouée, avocate, agrégée n'ont rien qui choque ; mais docteur a fait difficulté, et aussi professeur, ingénieur; croque-morte a l'air d'une funèbre plaisanterie. Le problème n'est pas simple. Dans beaucoup de cas, on s'en est tiré en ajoutant le mot femme : exposition des femmes peintres et sculpteurs. Ce n'est qu'un pis aller. Quelques formes se féminiseraient facilement : ingénieure, professeure, s'écriraient comme supérieure, mais doctoresse empêche docteure. Chefesse est horrible.

Ce qui augmente la difficulté, c'est que beaucoup de femmes croiraient n'avoir rien obtenu, si l'assimilation n'était pas complète. Elles veulent porter tout crus des titres d'hommes: Mademoiselle le Dr. Un tel [...]. (Brunot 1922 : 90) 
Les femmes et le langage selon Charles Bally : "des moments de décevante inadvertance"?

L'ouverture de Bally apparaît à nouveau par contraste ${ }^{18}$, notamment à travers les réactions suscitées par une féminisation particulière - chefesse ou chéfesse - dont la graphie varie selon les époques et les énonciateurs, mais ne cesse de susciter le commentaire. Ce lexème, hautement symbolique, est régulièrement convoqué à des fins d'emblématisation de l'impossible féminisation; il a été glosé d'innombrables fois et aujourd'hui encore provoque les passions des spécialistes comme des praticien-ne-s. Tandis que les premiers récusent la féminisation de chef au nom de son sens étymologique (caput, la tête), même oublié par le commun des sujets parlants, les seconds la rejettent pour des raisons esthétiques le plus souvent. Ainsi, en 1994, Henri Morier qualifie la forme "cheffe" - aussi souvent pratiquée que dénoncée en Suisse romande depuis une vingtaine d'années - de monstre "qui ne saurait être qu'un accident ou qui pourrait à la rigueur naître sous la plume d'un burlesque" (1994 : 94). Il n'est pas anodin que ce soit ce terme, plus que tout autre, qui suscite les commentaires enflammés. Cela montre bien que derrière la réflexion morphologique, c'est la question de pouvoir qui en réalité se profile.

Tandis que chefesse est rejeté sans appel par un nombre non négligeable de spécialistes et de sujets parlants, il est considéré de façon assez détachée par Bally comme "paraissant dur". Le recours au modalisateur "paraissant" atténue l'évaluation négative "dur" et la met à distance. Il semble donc que Bally ne prenne pas en charge le rejet de féminisations comme chéfesse au nom d'une éventuelle dureté, mais qu'il se contente de signaler le sentiment des sujets parlants. Damourette et Pichon sont encore plus tolérants puisqu'ils se limitent à signaler que "la langue parlée emploie couramment chefesse comme féminin du chef" (1927 : 316) sans évoquer de possibles réactions de rejet.

On notera au passage que l'article 503 (cf. citation $\mathrm{n}^{\circ} 38$ supra) ne figure pas moins de trois fois dans l'index de la dernière édition de Linguistique générale et linguistique française. Cet article est mentionné d'abord sous inconséquence (dans l'emploi des suffixaux féminins en fr.), ensuite sous féminin, et enfin sous formation des féminins, dont il constitue le seul renvoi. Un tel dispositif de mise en évidence tend à montrer qu'il s'agit là d'une problématique d'une certaine importance pour Bally, qui se montre cependant beaucoup moins engagé que d'autres contemporains, parmi lesquels on peut à nouveau mentionner Damourette et Pichon, qui, comme Brunot (citation $n^{\circ} 39$ ), dénoncent vivement le refus de féminisation, en particulier lorsqu'il émane des femmes elles-mêmes :

40. La facilité avec laquelle le français, soit par le procédé flexionnel, soit par le procédé suffixal, sait former des féminins différenciés devrait vraiment détourner les femmes adoptant des professions jusqu'à ces derniers temps exclusivement masculines de ridiculiser leurs efforts méritoires par des dénominations masculines écœurantes et grotesques, aussi attentatoires au génie de la langue qu'aux instincts les plus élémentaires de l'humanité. [...] Une plus juste conception de leur véritable place et de leurs légitimes aspirations, en même temps que le respect de la langue maternelle, devrait au contraire leur conseiller de renoncer au préjugé bizarre en vertu duquel

\footnotetext{
18 Pour une critique détaillée de la position de Brunot, cf. Baudino 2001 et Michard 2002.
} 
beaucoup d'entre elles croient recevoir une marque de mépris quand on leur donne un titre à forme féminine. A moins que leur féminisme ne soit une conception contre nature et la négation non de l'inégalité mais de la différence des sexes, cette prétention barbare va contre leur but même. Ne se rendent-elles pas compte que, bien au contraire, au point de vue social même, elles ne font, en laissant obstinément à leur titre sa forme masculine auprès de leur nom féminin et de leur appellation féminine de Madame ou Mademoiselle, que se proclamer elles-mêmes des monstruosités, et que, dans une société où il deviendra normal de les voir exercer les métiers d'avocat, de médecin, d'écrivain, il sera naturel qu'il y ait pour les femmes se livrant à ces métiers des dénominations féminines comme il y en a pour les brodeuses ou les cigarières? (Damourette \& Pichon 1927 : 321)

Damourette et Pichon défendent ici un point de vue général plutôt progressiste ${ }^{19}$ en ce qui concerne les métiers susceptibles d'être exercés par les femmes. Dans cet extrait, les deux linguistes articulent le point de vue idéologique et le point de vue lexical, en montrant que sur le plan linguistique la féminisation ne constitue aucunement un problème et que les barrières doivent être situées sur le plan de l'usage et des effets symboliques. A travers le choix de leurs exemples, les deux linguistes montrent bien qu'il n'y a que certains noms de métier - les métiers prestigieux - qui offrent des résistances à la féminisation. Les deux auteurs attirent l'attention sur le fait que la désignation au masculin - par son caractère irrégulier, atypique sous-entend une interrogation sur la légitimité du métier pour une femme.

La tendance progressiste de Damourette et Pichon a cependant des limites précises, puisqu'il n'est pas question d'interroger un certain partage des sexes ou la naturalité de rôles sexuels différenciés. Malgré tout, rien dans ces lignes ne laisse entendre que le métier de brodeuse ou de cigarière serait plus adéquat pour une femme que celui d'avocate ou de médecin(e). Il me semble donc nécessaire de nuancer, ici aussi, le jugement excessivement sévère de Claire Michard sur ces deux auteurs, dont elle glose pourtant, de façon très intéressante leur expression "adopter" des professions :

41. La mainmise sur les professions lucratives et / ou prestigieuses par les hommes et la lutte des femmes contre cette injustice sont énoncées comme des événements naturels, hors pratiques sociales. Pour énoncer la chasse gardée des hommes, on désembraye systématiquement et hypocritement leur agentivité : "fonctions", "domaines", "professions" "réservées aux hommes". "Réservés" par quelles pratiques sociales ? Ce biais dans la pensée et le langage peut aller jusqu'à la caractérisation de certaines professions comme intrinsèquement "masculines" : "professions masculines".

De la même façon, bien que cette "accession" s'étende sur une durée de soixante ans, il est impossible de présenter l'accès des femmes à certaines professions comme une course d'obstacles de très longue haleine et une lutte sociale jamais achevée : "les portent s'ouvrent", "les activités féminines pénètrent", "l'accession des femmes", "les femmes ont accès"... Seule l'expression "les femmes adoptant", les présente comme à l'origine de leur accès à certaines professions, mais le contexte est fortement hostile. "Adopter" élimine toute idée de difficulté et de combat; il suggère

19 Il faudrait évidemment débattre du point de vue différentialiste, voire essentialiste, qui semble animer Damourette et Pichon dans cette même citation. 
Les femmes et le langage selon Charles Bally : "des moments de décevante inadvertance"?

en outre une idée de "non-naturalité" (adopter un enfant), surtout lorsqu'il est associé à "profession masculine" : la "monstruosité" n'est pas loin. (Michard 2002 : 147)

Le texte de Damourette et Pichon, comme celui de Bally, est traversé de nombreuses tensions, qu'on ne saurait occulter, et qui ne font pas de ces auteurs des féministes au sens moderne du terme. Il est clair qu'il demeure dans leurs textes un fonds indéniable de patriarcat, plus ou moins manifeste, plus ou moins assumé, que l'on ne saurait nier, mais pas non plus hypertrophier.

S'exprimant également sur la question de la féminisation, Albert Dauzat (1877-1955), directeur à l'Ecole des Hautes Etudes, envisage la langue comme un frein au changement social, à l'instar de Bally :

42. Le féminisme dans ses conquêtes a marché à pas de géant; la grammaire, cette tardigrade, ne l'a suivi qu'à contrecœur. De sorte qu'à l'heure actuelle il y a disproportion entre les besoins et les moyens. (Bally 1955 : 99)

Comme Damourette et Pichon, mais beaucoup plus explicitement que son collègue genevois, Dauzat se prononce en faveur de la féminisation :

43. La femme qui préfère pour le nom de sa profession le masculin au féminin accuse par là même un complexe d'infériorité qui contredit ses revendications légitimes. Dérober son sexe derrière le genre adverse, c'est le trahir. Proclamer la supériorité du masculin, madame le docteur, c'est reconnaître implicitement la supériorité du mâle dont le masculin est l'expression grammaticale. (Dauzat 1955 : 100)

On notera au passage que, comme Bally, Dauzat constate que chéfesse passe mal (1955: 106). Tandis que ce dernier milite clairement pour la féminisation des titres, fonctions, etc., Bally se contente de stigmatiser certaines inconséquences. On notera cependant que le linguiste genevois ne recourt guère à des formulations du type le féminin de tel substantif masculin qui donnent à penser que le masculin constitue la forme de base et qui révèlent, selon Claire Michard, sous la prétention universaliste, une perspective nettement androcentrique :

44. Le modèle sous-jacent aux expressions "femelle du cheval", "femelle de l'oiseau" est celui de la définition sociale des femmes : femmes des hommes, autrement dit, n'appartenant pas (au sens logique) à l'espèce "homme", mais appartenant (au sens juridique et politique) aux hommes mâles de l'espèce. (Michard $2002: 50$ ) 


\section{$4 \quad$ Le masculin l'emporte : un sentiment de barbarisme}

Autre point volontiers discuté par la linguistique générique, le nécessaire accord au masculin en cas de noms mixtes sur le plan du genre grammatical. A nouveau Bally développe un point de vue étonnamment progressiste :

45. [...] un adjectif peut qualifier deux substantifs. Alors l'habitude des rapports par juxtaposition amène à attribuer l'adjectif au second substantif seulement : "une dame et une demoiselle très aimable(s)". L'orthographe tranche la question, mais l'audition laisse des doutes.

Si les deux substantifs sont de genres différents et que l'adjectif distingue le masculin et le féminin, la difficulté est encore plus grande : quand l'adjectif suit le substantif féminin, on a le sentiment d'un barbarisme : "un monsieur et une dame élégants", "La musique devient besoin et denrée internationaux" (Nouv. Litt., 28-3-31). Il n'est pas rare qu'on s'en tire par un accord unilatéral illogique : "Tout l'ensemble rayonne autour de ce sommet avec un ordre et une symétrie parfaite" (Grammont, Le Français moderne, 6, p. 51).

Si l'on renverse les termes ("une dame et un monsieur élégants"), on pense instinctivement que le second substantif est seul qualifié. Cf. "Manuel de langue et de style français", "Le langage et la politesse reposent en grande partie sur des conventions et des tabous sociaux".

L'amphibologie est moindre quand les deux substantifs forment un collectif (146) : "les parents et amis invités à la noce; les mandats et colis postaux"; mais dans "grammaire et travaux spéciaux relatifs aux langues slaves", spéciaux semble se rapporter à travaux et relatifs aux deux substantifs ! (Bally LG et LF, $\mathrm{n}^{\circ} 431: 265$ )

A la différence d'un certain nombre de ses collègues, Bally ne considère pas que le fait d'achever une énumération ou un couple mixte par l'élément masculin résolve le problème ${ }^{20}$. A son sens, quel que soit l'ordre de la combinaison \{GNMasc. + GNFém. > adj. Masc. \} ou \{GNFém. + GNMasc. > adj. Masc.\}, l'allocutaire a le sentiment que l'adjectif masculin ne qualifie que le groupe nominal masculin. On ne lève donc pas la difficulté en s'arrangeant pour que le dernier terme de l'association ou de l'énumération soit masculin, ce qui a pour effet un moindre sentiment de "barbarisme". Une telle astuce ne résoudrait pas tous les problèmes et surtout elle n'annulerait pas le sentiment que l'adjectif porte, en fait, exclusivement sur le dernier terme de l'énumération. L'accord de proximité, qui constitue une pratique assez fréquente et qui deviendra l'option préférentielle de certains guides de rédaction non discriminatoires (cf. Moreau 2001: 37), n'offrirait pas une solution satisfaisante. Pour éviter l'effet particularisant, la seule solution est que les divers termes de l'énumération "forment un collectif" sur le plan sémantique et/ou syntaxique.

\footnotetext{
20 Il s'agit là d'une stratégie qui consiste à appliquer, sans en avoir l'air, la règle de proximité, qui veut que l'on accorde l'adjectif avec le dernier terme d'une énumération. Rappelons que cette règle a été rendue caduque au milieu du XVIIe siècle par Vaugelas au nom de la plus grande noblesse du masculin. Pour mémoire, Claude Vaugelas est le principal instigateur de la suprématie de la règle du "masculin l'emporte", règle justifiée par la raison patriarcale : "[...] pour une raison qui semble être commune à toutes les langues, que le genre masculin étant le plus noble, doit prédominer toutes les fois que le masculin et le féminin se trouvent ensemble" et cela même si "l'oreille a de la peine à s'y accommoder" (Vaugelas 1934).
} 
Les femmes et le langage selon Charles Bally : "des moments de décevante inadvertance"?

Une fois de plus Bally s'oppose à une approche strictement formelle ou grammaticalisante, et même prescriptive; il prend acte de la réception des sujets parlants, des effets produits par certains choix ou combinaisons sur ces derniers.

\section{De l'influence du sexe sur l'interlocution}

L'essentiel des réflexions "génériques" ou "sexuées" de Bally relève d'une perspective que l'on peut qualifier globalement de systémique, même si la conception ou la pratique du système est certainement plus large chez Bally que chez d'autres linguistes. En effet, sur ce point aussi, ce linguiste a ouvert considérablement le système sur l'usage, voire sur les sujets parlants. Comme cela a été évoqué au début de cet article, Bally se concentre essentiellement sur une perspective énonciative et s'intéresse plus rarement à la dimension conversationnelle. Aussi n'est-il pas surprenant que l'éventuelle spécificité de comportements conversationnels masculins et féminins ne soit pas abordée. En revanche, on peut s'étonner que Bally aborde la question sous l'angle de l'appartenance sexuelle de l'allocutaire ou du délocuté, qui, à l'en croire, peut exercer une influence sur le discours du locuteur :

46. Mais ce n'est pas tout : la présence ou la simple représentation mentale d'autres personnes peut exercer une action coercitive sur notre langage. Ainsi, en parlant avec quelqu'un, ou en parlant de lui, je ne puis m'empêcher de me représenter les relations particulières (familières, correctes, obligées, officielles) qui existent entre cette personne et moi ; involontairement je pense, non seulement à l'action qu'elle peut exercer sur moi, je me représente son âge, son sexe, son rang, le milieu social auquel elle appartient; toutes ces considérations peuvent modifier le choix de mes expressions et me faire éviter tout ce qui pourrait détonner, froisser, chagriner. (Bally LV : 21. Je souligne.)

Dans cet extrait, Bally passe en revue un certain nombre de facteurs d'influence, parmi lesquels on trouve les variables traditionnelles que sont l'âge, le rang ou le milieu social. A ces facteurs habituels de variation, Bally ajoute la variable du sexe de l'allocutaire, qui détermine plus ou moins la configuration du discours de l'instance locutrice. Il faut souligner que ce sont moins les caractéristiques effectives de l'allocutaire qui exercent une influence que leur représentation dans l'univers de croyance de l'instance locutrice. On soulignera également que le délocuté lorsqu'il renvoie à des personnes peut influencer l'énonciation et l'énoncé de l'instance locutrice. Le délocuté est alors considéré comme un élément signifiant de la situation de communication.

\section{$6 \quad$ Une langue spéciale : le parler féminin ?}

L'intérêt de Bally pour la diversité linguistique l'amène malgré tout à envisager l'existence potentielle d'un parler féminin, qui ne constituerait qu'une forme de l'extraordinaire diversité 
linguistique, une langue spéciale parmi ces autres langues spéciales que sont la langue administrative, la langue des sports, la langue littéraire, la langue technique, etc. :

47. On nous dit, par exemple que, chez les Cafres [tribu de la partie méridionale de l'Afrique], les femmes parlent entre elles une autre langue que celle qu'elles parlent avec les hommes; c'est un fait analogue à ce que nous avons appelé des langues spéciales, et l'origine de cette distinction est purement sociale ; est-on bien sûr que le cas soit très différent de celui d'un huissier français qui, en famille, parle comme tout le monde, mais, pour libeller une minute, écrit un charabia que beaucoup de ses compatriotes sont incapables de comprendre ? (Bally LV : 51. Je souligne.)

On notera toutefois que si Bally est prêt à accepter l'existence d'une langue spéciale féminine chez les Cafres, cela ne l'amène pas à s'interroger sérieusement sur l'existence d'une telle variante en français. Joseph Vendryes, avec lequel Bally entretenait des liens étroits et dont il connaissait bien les travaux, se montre plus audacieux. Dans un passage qui développe des considérations très proches de Bally, Vendryes prend en considération la langue administrative, la langue religieuse, la langue médicale, l'argot des malfaiteurs, la langue des sorciers, la langue des chercheurs de camphre, celle des chercheurs d'or et, presque tout à la fin, la langue des femmes :

48. Une des spécialisations les plus fréquentes résulte de la différence des sexes. Les femmes n'emploient pas le même langage que les hommes; même lorsqu'elles comprennent les mots qu'emploient ceux-ci, elles n'ont jamais le droit de les prononcer. Il y a deux vocabulaires différents, exactement parallèles, de sorte que chaque objet a deux noms, suivant le sexe de celui qui parle. Chez les Caraïbes par exemple, les hommes parlent caraïbe, mais les femmes parlent arowak. Parfois les différences de langue recouvrent des différences de classe sociale. Chez les naturels de Java, le supérieur parle à son inférieur en langue ngoko, mais l'inférieur répond en langue kromo. (Vendryes $1968: 284$ )

Vendryes développe un peu plus sa réflexion que Bally, mais semble se garder d'aller trop loin sur ce terrain. Ces propos appellent plusieurs remarques. Tout d'abord, Bally ne met pas en doute l'analyse, mais il ne s'y arrête pas non plus. Il oppose à de telles considérations un silence prudent, qui contraste singulièrement avec les propos développés par le linguiste danois Otto Jespersen (1860-1943), également connu de Bally. Jespersen, dans un livre (Language 1922) qui subira les justes foudres de la linguistique générique, s'arrête longuement sur la problématique de "la langue des femmes", qu'il va tantôt dénigrer comme étant restreinte sur le plan lexical, simpliste sur le plan syntaxique, etc., et tantôt encenser pour sa tendance standardisatrice, voire conservatrice. Bally connaissait probablement les travaux de Jespersen, mais ne les relaie pas, du moins pas explicitement. On trouve cependant dans des écrits latéraux de Bally des considérations analogues, qui voient dans le discours des femmes un discours naturellement conservateur :

49. La mère est donc prédestinée à cette initiation [à la langue maternelle], et elle possède en elle tout ce qu'il faut pour faire un bon professeur de langue. Aujourd'hui, comme dans toutes les époques troublées, la langue est menacée de déséquilibre ; elle change trop rapidement, se disloque, perd 
Les femmes et le langage selon Charles Bally : "des moments de décevante inadvertance"?

de son unité. Or, la femme a, par instinct, le sens de la tradition, le respect de l'usage admis et transmis, le goût inné de la pureté, qui lui fait éviter tout ce qui est vulgaire et bas. (Bally, L'Illustré, 1937, cité par Hellman 1988 : 90-91)

Ces lignes nous incitent à ne pas exagérer le caractère novateur, voire féministe, de Bally, qui ici attribue le sens de la tradition de la femme à son instinct et non pas à son éducation ni à son rôle social.

Dignes de leur époque, ces propos ont malgré tout de quoi surprendre de la part d'un homme qui s'est, ailleurs, violemment élevé contre ce sentiment récurrent et non fondé de dégénérescence du français. En faisant des femmes les gardiennes du trésor de la langue et les institutrices de leur progéniture, Bally soutient des idées défendues par un nombre non négligeables de linguistes (Jespersen mais aussi Gauchat, Gilliéron et bien plus tard Labov) ou de chroniqueurs du langage, comme Robert de Traz, dont il était par ailleurs un adversaire farouche :

50. Dans une ville comme Genève où les pédagogues pullulent, il est extraordinaire de constater le peu d'importance qu'on donne à l'enseignement de notre langue maternelle. [Ou bien les enfants] se fâchent et se taisent d'un air renfrogné. Ou bien ils parlent un effroyable charabia, mêlé d'argot genevois, d'argot parisien, de termes impropres, de termes fabriqués par eux-mêmes. [...] Ainsi cette jeune femme qui s'écriait :"Hier, on a fait une pistée en rase." - ce qui signifiait : "Nous avons été nous promener à la campagne." Plaignons les rejetons de cette dame: ils seront ridicules et malheureux. (de Traz, "Notre langue maternelle", Journal de Genève, 30 octobre 1929)

\section{$7 \quad$ Bally, un féministe avant l'heure ?}

Il apparaît que Bally, comme de nombreux savants de son époque, a abordé les questions qui deviendront celle de la linguistique générique. Si les positions de Bally ne se distinguent pas toujours par leur caractère détaillé, elles se singularisent cependant par leur étendue comme par leur acuité. En effet, Bally a abordé l'ensemble des domaines aujourd'hui envisagés en linguistique générique, de façon relativement neuve et "féministe" à la différence de certains de ses collègues. Bally, mais aussi Damourette et Pichon ou Dauzat, nous incitent à émettre un bilan quelque peu plus nuancé que celui de Claire Michard :

51. Pendant cette période des années vingt aux années soixante-dix, et malgré les différences théoriques importantes, on peut constater l'accord des linguistes sur les points suivants: morphologiquement, le genre féminin est un genre dérivé ; syntaxiquement, le genre masculin domine le genre féminin ; sémantiquement, le genre féminin a une moindre valeur que le genre masculin; le sens est défini par l'application des noms aux référents extralinguistiques non-définis sociologiquement; le trait sémantique de sexe est inhérent au genre féminin et il ne semble pas, ou moins, l'être au genre masculin. En outre, la contradiction entre l'affirmation de la symétrie sémantique, au sexe près, et certaines formulations qui présentent la femelle d'une espèce comme 
la femelle ou la "femme" du mâle de l'espèce, ainsi que le traitement séparé de la forme et du sens (description asymétrique de la structure formelle et description symétrique de la structure sémantique), produisent un double discours mêlant raisonnement logique et effet idéologique du rapport d'appropriation des femmes par le les hommes, rapport conceptualisé par Guillaumin et dénommé "sexage". (Michard 2002 :133)

Une partie des considérations de Bally peut s'expliquer par le fait que pour ce francophone de la périphérie, la langue a toujours été multiple et mouvante. En revanche, pour de nombreux linguistes hexagonaux, la langue n'est pensable que dans l'unité et la permanence. Il s'agit là je crois d'un reste de jacobinisme linguistique. On ne vit pas impunément dans un pays qui a imposé de façon très active et parfois violente le dogme monolingue. Les conceptions de la Révolution française ont laissé des traces très vivaces, tant dans les pratiques - ou plutôt les représentations - sociales, que dans les pratiques scientifiques autorisées.

En revanche, la Suisse - alémanique en particulier - se caractérise par une acceptation plus élevée d'une forte diversité de pratiques. Il n'est pas étonnant que Bally, linguiste suisse, ait été très ouvert à la diversité des langues et à la variation linguistique.

52. L'idiome vulgaire et parlé continue sa marche, d'autant plus sûre qu'elle est souterraine; il coule comme une eau vive sous la glace rigide de la langue écrite et conventionnelle; puis un beau jour la glace craque, le flot tumultueux de la langue populaire envahit la surface immobile et y amène de nouveau la vie et le mouvement. (Bally LV : 13)

On peut souligner au passage que la Suisse a fourni à la France de nombreux dialectologues, qui ne pouvaient être formés en son sein, tant le dogme du monolinguisme était encore prégnant au début du siècle. La France a vécu sous la bannière du monolinguisme incontournable, gage de l'unité nationale. Ce monolinguisme se devait d'être sans faille, y compris sur le plan scientifique. Pendant de nombreuses décennies, l'hexagone a peiné à reconnaître la diversité des langues et la variation linguistique. L'acceptation de la diversité ne pouvait se faire que parcimonieusement et au prix d'une articulation hiérarchique des différentes "langues" envisagées, qui continuerait de reconnaître à une variété, en l'occurrence écrite et littéraire, sa supériorité, sa valeur de norme ${ }^{21}$.

Bally se singularise alors doublement, d'abord par une reconnaissance de la diversité et ensuite par sa contestation de la hiérarchie institutionnelle, en faisant par exemple de la langue littéraire une langue spéciale parmi d'autres, comme la langue administrative par exemple.

53. [...] La langue littéraire a surtout une valeur sociale, c'est un symbole de distinction, de bonne tenue intellectuelle, d'éducation supérieure; la linguistique ne peut l'envisager autrement que comme l'une de ces langues spéciales [...]. A ce titre, elle a sa place - place d'honneur, il est vrai -

21 Pour un élargissement du débat à d'autres domaines du savoir (littérature, pédagogie, etc.), voir notamment Durrer 1990 et Meizoz 2001. 
Les femmes et le langage selon Charles Bally : "des moments de décevante inadvertance"?

aux côtés de la langue administrative, de la langue scientifique, de la langue des sports, etc. (Bally LV : 28)

De façon emblématique, on notera que, jusqu'à il y a peu, le recours à une prononciation standard était à peine discuté et expliqué par la rédaction du Robert. Voici notamment ce qu'on pouvait lire dans les pages d'introduction du Petit Robert en 1979 :

54. D'une manière générale, nous n'avons noté pour chaque forme écrite qu'une seule prononciation. Nous avons pris comme norme la conversation soignée du Parisien cultivé. (LUCOT, Aliette., REYDebove, Josette, 1979, "Principes généraux de la transcription phonétique", Le Petit Robert, p. XXII.)

La norme - parisienne, bourgeoise et masculine, en l'occurrence - était donnée comme une évidence indiscutable. Nous avons là affaire au phénomène que les études génériques décrivent depuis de nombreuses années :

55. Si le sujet philosophant [ou "linguistiquant"] se définit implicitement comme sujet masculin, mais exempté dans le même temps de sa partialité d'être sexué, on ne s'étonnera pas de la position occupée par les femmes dans son dispositif de pensée. (Collin, Pisier \& Varikas 2000 : 20-21)

Ce type de présupposé n'est pas uniquement le fait du discours parascientifique, on le trouve au cœur même des discours scientifiques les plus en vue, comme celui de William Labov, si souvent présenté comme le père de la sociolinguistique :

56. Vers 1960, en dépit d'une quantité de travaux sociolinguistiques, l'apparition d'une linguistique attachée à la réalité sociale était encore, semblait-il, une perspective lointaine. A présent, quoique l'intuition nous couvre encore de son ombre, il ne paraît plus nécessaire de discuter de ce qu'est ou n'est pas la linguistique. On s'aperçoit de plus en plus que le fondement de la connaissance intersubjective en linguistique ne se trouve pas ailleurs que dans la parole - le langage en tant qu'il est utilisé quotidiennement par les membres de l'ordre social, en tant que moyen de communication grâce auquel ils discutent avec leurs femmes, plaisantent avec leurs amis et trompent leurs adversaires. (LABOv, William, 1976, Sociolinguistique, Paris, Minuit : 37. Je souligne.) (Cité par Baudino 2001 : 185)

Il s'agit là d'un cas exemplaire. Au moment où Labov introduit la nécessaire prise en compte de la réalité sociale et de sa diversité, il en exclut, implicitement, les femmes. L'ouverture du dispositif ne concerne une fois de plus que le monde masculin, donné - plus que pensé comme évidemment universel.

Tandis que le point de vue latent, indéniablement normatif du discours linguistique, a été démontré et critiqué par Alain Berrendonner notamment, dans son ouvrage L'éternel grammairien (1982), l'éternel masculin ou "androcentrisme" du discours linguistique doit encore être mis au jour et problématisé. Pour l'heure, la plupart des linguistes ne sont pas conscient-e-s de cette orientation et manifestent un "gender blindness". 
Une prise de conscience et une reconnaissance grandissantes de la variation linguistique apparaissent lentement dans le monde franco-français. En guise d'indice de ce changement, on peut mentionner les modifications récemment survenues dans la préface du Robert :

57. Dans le cas de réalisations phonétiques multiples, nous avons choisi de noter une seule des variantes possibles, de préférence la plus conforme à la prononciation récente des locuteurs urbains éduqués d'Ile-de-France et de régions voisines, en espérant ne pas choquer les utilisateurs d'usages plus anciens, ruraux ou de régions où subsiste soit un bilinguisme, soit l'influence d'une autre langue ou de dialectes (par ex. Occitanie, Bretagne, Alsace...). Il y a cependant une exception à cette préférence pour la transcription unique : les emprunts. (LUCOT-SARIR, Aliette, ReY-Debove, Josette, 2000, "Choix de présentation de la phonétique", Le Petit Robert, p. XXI.)

L'ouverture, nécessaire et bienvenue, ne franchit toutefois pas encore certaines limites. Parmi les diverses variétés reconnues très tôt par Bally, il en est une qu'une partie non négligeable du monde linguistique franco-français peine, encore aujourd'hui, à prendre en compte, il s'agit de la parole des femmes, et plus largement de la prise en compte du facteur "femme" ou plus universellement encore du facteur "sexué". Les problématiques rassemblées parfois sous la désignation de "Language and Gender" figurent également dans les publications de Bally. Il s'agit là d'un axe de réflexion qui n'a guère été mentionné à propos de Bally et qui mérite d'être mis en lumière, non pas pour faire de Bally un féministe avant l'heure, mais simplement, parce qu'il semble important de documenter l'histoire d'une problématique dans un champ disciplinaire donné. Cet article se conçoit donc comme une contribution à cette perspective. Travaillant dans une orientation dont la reconnaissance institutionnelle n'est pas encore complètement acquise, il me semblait important de montrer que cette question du rapport des femmes et du langage en français est une question qui mérite d'être posée, qu'il serait intéressant pour la linguistique de procéder à une histoire critique de cette thématique, qui aurait pour objet de voir si elle est abordée, dans quel contexte, avec quels résultats, etc. On serait alors peut-être surpris-e de constater que cette problématique est envisagée par un grand nombre d'auteur-e-s majeur-e-s, dont Charles Bally.

Sans exagérer l'importance de ces considérations dans le cadre d'une œuvre dont les lignes de force étaient ailleurs, sans non plus y voir une orientation uniment féministe, il s'agit malgré tout de reconnaître leur présence et leur diversité. On ne peut à tant de considérations, plus ou moins développées, répondre par une réaction épidermique à la façon d'un Henri Morier et considérer que Bally, à l'instar de Ferdinant Brunot, se serait laissé aller à de simples moments de "décevante inadvertance" :

58. On ne peut donc créer des féminins de profession par simple adjonction d'un -e, en dépit de ce que F. Brunot, dans un moment de décevante inadvertance, déclarait naguère : "Quelques formes se féminiseraient facilement : ingénieure, professeure s'écriraient comme supérieure." (La Pensée et la langue, $3^{\mathrm{e}}$ éd., 1953, p. 90.) Non, cela ne suffit pas, nous le regrettons pour la mémoire du grand linguiste. (Morier 1993 : 87) 
Les femmes et le langage selon Charles Bally : "des moments de décevante inadvertance"?

Ne pourrait-on pas, tout au contraire, dire que les propos de Bally sur les rapports entre les femmes, les hommes et le langage constituent, à bien des égards, des moments de fulgurante lucidité ?

\section{Bibliographie}

Amacker, René (1991): "Charles Bally et La 'stylistique"'. In: Huot, Hélène (éd.) (1991): La grammaire française entre comparatisme et structuralisme (1870-1960). Paris: 115-154.

Apothéloz, Denis (2002): La construction du lexique français. Paris.

Arrivé, Michel / Gadet, Françoise / Galmiche, Michel (1986): La grammaire d'aujourd'hui. Paris.

Bakhtine, Mikhail (1977): Le marxisme et la philosophie du langage. Paris [1929].

Bally, Charles (1922): "La pensée et la langue". Bulletin de la société linguistique de Paris 22-23: 116-137.

Bally, Charles (1930): La Crise du français. Notre langue maternelle à l'école. Paris \& Neuchâtel.

Bally, Charles (1940): "L'arbitraire du signe. Valeur et signification". Le Français moderne. Paris: 193-206.

Bally, Charles (1965): Linguistique générale et linguistique française. Paris: 410 p. Deuxième édition refondue et augmentée: Berne 1944. Dernière édition légèrement corrigée 1965.

Bally, Charles (1977): Le langage et la vie. Troisième édition refondue et augmentée: Genève [1952].

Baudino, Claudie (2001): Politique de la langue et différence sexuelle. La politisation du genre des noms de métier. Paris.

Bergvall, Victoria L. (1999): "An Agenda for Language and Gender Research for the Start of the New Millennium". Linguistik online 2, 1/99.

Berrendonner, Alain (1982): L'éternel grammairien. Etude du discours normatif. Berne \& Francfort.

Bonnard, Henri (1972): "Genre". In: Grand Larousse de la langue française en sept volumes. Vol. 3. Paris: 2201-2211.

Brunot, Ferdinand (1922): La pensée et la langue. Paris.

Chevalier, Jean-Claude (1991): "Ferdinand Brunot. La pensée et la langue". In: Huot, Hélène: La grammaire française entre comparatisme et structuralisme (1870-1960). Paris: 155200.

Chevalier, Jean-Claude / Blanche-Benveniste, Claire / Arrivé, Michel / Peytard, Jean (1974): Grammaire Larousse du français contemporain. Paris.

Collin, Françoise / Pisier, Evelyne / Varikas, Eleni (2000): Les femmes de Platon à Derrida. Anthologie critique. Paris.

Dauzat, Albert (1955): Le guide du bon usage. Les mots, les formes grammaticales, la syntaxe. Paris.

Darmsteter, Arsène (1930): Cours de grammaire historique de la langue française. Paris. 
Damourette, Jacques / Pichon, Edouard (1970): Des mots à la pensée : essai de grammaire de la langue française [1911-1940], 8 vol. Paris.

Durrer, Sylvie (1990): "Les droits de l'oral et des dialectes dans le roman du XIXème siècle". Equinoxe, $\mathrm{n}^{\circ}$ 3. Lausanne: 79-99.

Durrer, Sylvie (1998): Introduction à la linguistique de Charles Bally. Paris. (= coll. Sciences des discours).

Hellmann, Wilhelm (éd.) (1988): Charles Bally. Unveröffentlilchte Schriften. Comptes rendus et essais inédits. Bonn.

Huot, Hélène (1991): La grammaire française entre comparatisme et structuralisme (18701960). Paris.

Jespersen, Otto (1922): "The Woman" (chap. XIII). In: Jespersen, Otto: Language. Its Nature, Development and Origin. London.

Khaznadar, Edwige (1993): "Pour une première : la dénomination de la femme dans l'actualité. Dichotomie, affixation et alternance". Cahiers de Lexicologie 63: 143-169.

Khaznadar, Edwige (2000): "Masculin et féminin dans la dénomination humaine". Revue de linguistique française 2: 141-170.

Lakoff, Robin (1975): Language and Woman's Place. New York.

Meizoz, Jérôme (2001): L’âge du roman parlant (1919-1939) : écrivains, critiques, linguistes et pédgagogues. Genève.

Michard, Claire (2002): Le sexe en linguistique. Sémantique ou zoologie ? Les analyses du genre lexical des années 1920 aux années 1970. Paris.

Moreau, Thérèse, et al. (1991): Dictionnaire féminin-masculin des professions, des titres et des fonctions. Genève.

Moreau, Thérèse, et al. (2001): Ecrire les genres. Guide romand d'aide à la rédaction administrative et législative épicène. Etat de Genève-DF-SPPEgalité-CDLE.

Morier, Henri (1993): "Ah! la belle professeure! - Où nous mène le désir de sexuisemblance généralisée". Cahiers Ferdinand de Saussure 47: 83-105.

Nyrop, Kristoffer $\left(1936^{2}\right)$, Grammaire historique de la langue française. Paris.

Planté, Christine (2001): "De quelques discours sur l'e muet". In: Stistrup Jensen, Merete (éd.): Nature, langue, discours, Lyon: 47-63. (= coll. Cahiers Masculin / Féminin).

Pusch, Luise F. (1984): Das Deutsche als Männersprache. Aufsätze und Glossen zur Feministischen Linguistik. Frankfurt/Main.

Riegel, Martin / Pellat, Jean-Christophe / Rioul, René (1994): Grammaire méthodique du français. Paris.

Spender, Dale (1980): Man Made Language. Londre.

Traz, Robert de (1929): "Notre langue maternelle". Le Journal de Genève, n²97, 30 octobre 1929.

Trömel-Plötz, Senta (ed.) (1984): Gewalt durch Sprache. Die Vergewaltigung von Frauen in Gesprächen. Frankfurt/Main.

Vaugelas, C.F. de (1934): Remarques sur la langue française [1647]. Paris.

Vendryes, Joseph (1968): Le langage. Introduction linguistique à l'histoire. Paris [1923]. (=L'Evolution de l'humanité) 
Les femmes et le langage selon Charles Bally : "des moments de décevante inadvertance"?

Wüest, Jakob (éd.) (1997): Les linguistes suisses et la variation linguistique. Basel \& Tübingen.

Yaguello, Marina (1978): Les mots et les femmes. Paris. 